\title{
Package 'netglm'
}

June 25, 2021

Type Package

Title netglm - generalized linear models for network data

Version 0.1 .0

Author Timon Elmer

Maintainer Timon Elmer <timon.elmer@gmail.com>

Description Estimates generalized linear models for network data based on the Quadratic Assignment Proccedure (Krackhardt, 1988). This package allows to investigate associations between characteristics of dyads in networks (e.g., the level of homophily between two actors) and a binary or continuous tie variable (e.g., friendship, amount of time spent together). One unique feature of this package is that it allows to estimate multi-group QAP models, where multiple networks are analyzed simultaneously (e.g., networks of multiple classrooms). Furthermore, parallel processing is implemented.

License GPL $(>=3)$

Encoding UTF-8

LazyData true

RoxygenNote 7.1.1

Suggests testthat

Imports ggplot2

\section{R topics documented:}

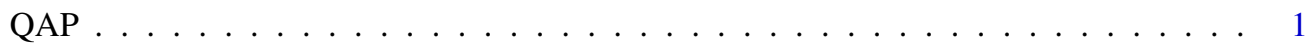

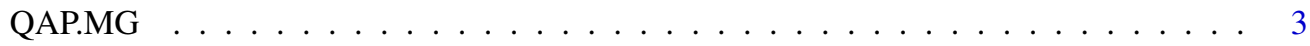

$\begin{array}{lr}\text { Index } & 6\end{array}$

QAP MRQAP for unnested data

\section{Description}

Estimates a Multiple Regression Quadratic Assignment Proccedure model (MRQAP; Krackhardt, 1988). MRQAPs allow investigating associations between characteristics of dyads in networks (e.g., the level of homophily between two actors) and a binary or continuous tie variable (e.g., friendship, amount of time spent together). 
Usage

QAP $(d v$, iv1, iv.names, mode $=$ "yQAP", samples $=1000$, diag $=F$, directed $=F)$

\section{Arguments}

$\mathrm{dv}$

iv1

iv.names

mode

samples

diag

directed

iv.list.per

family

round. to

$\mathrm{cpu}$

logfilename

verbose

global.deltas

return.perms a matrix with $\mathrm{n} *$ m dimensions and cells indicating the presence of a tie $(1=$ tie, $0=$ no tie for binary variables) or the weight of a tie (for continous tie variables) charcterizing the dependent variable

a list of matrices with $\mathrm{n} * \mathrm{~m}$ dimensions characterizing the independent variables names of the independent variables for the output object

permutation method to be applied. default is "yQAP" for permuting the Y / dv variables."dspQAP" applies Dekker's semi partialing method (Dekker, Krackhard, \& Snijders, 2007)

number of permutations, default is 1000 .

boolean for using the diagonal values of matrices in the estimation. default is FALSE

"directed" if the dependent network is directed (ties from A to B and B to A are possible), "undirected" if the dependent network is undirected (ties from A to B are identical to B to A). Default is "directed".

lists in the ivs argument are should be nested by group and independent matrices, if this is not the case (grouped by independent matrices and then groups) the argument iv.list.per $=$ "iv" can be used to restructure the data.

family of the generalized linear model. default is "gaussian" for continuous dependent varaibles. F or binday dependent variables "binomial" is advised.

numeric, numer of digits in output table

number of cpu's to be used for estimation, default is 1

name of log file printing intermediate reports during the estimation procedure.

reports of what is happening under the hood during the call of the function, default is TRUE

during "dspQAP" estimation, should global or local delta values be used. default is TRUE

should permuted networks be part of the output? default is FALSE

\section{References}

Dekker, D.; Krackhardt, D.; Snijders, T.A.B. (2007). "Sensitivity of MRQAP Tests to Collinearity and Autocorrelation Conditions." Psychometrika, 72(4), 563-581.

Krackhardt, D. (1987). “QAP Partialling as a Test of Spuriousness.” Social Networks, 9 171-186.

Krackhardt, D. (1988). "Predicting With Networks: Nonparametric Multiple Regression Analyses of Dyadic Data.” Social Networks, 10, 359-382.

\section{See Also}

QAP.MG 


\section{Examples}

\# create test data \#

inspired by the example funciton in sna: :netlm

ivnet1<-sna: : rgraph $(20,4)$

dv1<-ivnet1 $[1,]+,4 *$ ivnet $1[2,]+,2 *$ ivnet1 $[3,$,$] \quad \# Note that the fourth graph is unrelated$ $\mathrm{dv} 1<-\mathrm{dv} 1+\operatorname{rnorm}(400$, mean $=1, \mathrm{sd}=1)$

iv1 <- $\operatorname{list}(\operatorname{ivnet} 1[1,],, \operatorname{ivnet} 1[2,],, \operatorname{ivnet} 1[3,],, \operatorname{ivnet} 1[4,]$,

QAP.MG(list(dv1), list(iv1), iv.names = c("intercept", paste0("IV",1:4)), samples = 3000)

\section{Description}

Estimates a MRQAP model taking the multilevel/grouped nature of the into account. An application and detailed description of the multigroup extension of MRQAP can be found in Elmer and Stadtfeld (2020).

\section{Usage}

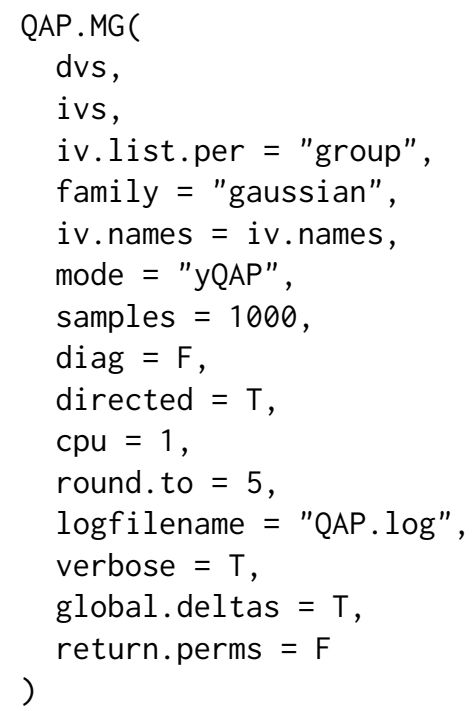

\section{Arguments}

dvs

a list of matrices where each matrix represents the dependent network of one group. Cells of the matrix indicating the presence of a tie $(1=$ tie, $0=$ no tie for binary variables) or the weight of a tie (for continuous tie variables) characterizing the dependent networks

ivs a list of lists with where each sets of independent matrices are grouped by group and then independent variables. If the list is organized per independent network the argument iv.list.per $=$ "iv" can be used to restructure the data.

iv.list.per lists in the ivs argument are should be nested by group and independent matrices, if this is not the case (grouped by independent matrices and then groups) the argument iv.list.per $=$ "iv" can be used to restructure the data. 


\begin{tabular}{|c|c|}
\hline family & $\begin{array}{l}\text { family of the generalized linear model. default is "gaussian" for continuous } \\
\text { dependent varaibles. F or binday dependent variables "binomial" is advised. }\end{array}$ \\
\hline iv.names & names of the independent variables for the output object \\
\hline mode & $\begin{array}{l}\text { permutation method to be applied. default is "yQAP" for permuting the Y / dv } \\
\text { variables."dspQAP" applies Dekker's semi partialing method (Dekker, Krack- } \\
\text { hard, \& Snijders, 2007) }\end{array}$ \\
\hline samples & number of permutations, default is 1000 . \\
\hline diag & $\begin{array}{l}\text { boolean for using the diagonal values of matrices in the estimation. default is } \\
\text { FALSE }\end{array}$ \\
\hline directed & $\begin{array}{l}\text { "directed" if the dependent network is directed (ties from A to B and B to A are } \\
\text { possible), "undirected" if the dependent network is undirected (ties from A to B } \\
\text { are identical to B to A). Default is "directed". }\end{array}$ \\
\hline cpu & number of cpu's to be used for estimation, default is 1 \\
\hline round. to & numeric, numer of digits in output table \\
\hline logfilename & name of log file printing intermediate reports during the estimation procedure. \\
\hline verbose & $\begin{array}{l}\text { reports of what is happening under the hood during the call of the function, } \\
\text { default is TRUE }\end{array}$ \\
\hline global.deltas & $\begin{array}{l}\text { during "dspQAP" estimation, should global or local delta values be used. default } \\
\text { is TRUE }\end{array}$ \\
\hline & should permuted networks be part of the output? default is FALSE \\
\hline
\end{tabular}

\section{References}

Dekker, D., Krackhardt, D., \& Snijders, T.A.B. (2007). "Sensitivity of MRQAP Tests to Collinearity and Autocorrelation Conditions." Psychometrika, 72(4), 563-581.

Elmer, T., \& Stadtfeld, C. (2020). "Depressive symptoms are associated with social isolation in face-to-face interaction networks". Scientific Reports, 1-12. https://doi.org/10.1038/s41598-02058297-9

Krackhardt, D. (1987). “QAP Partialling as a Test of Spuriousness.” Social Networks, 9 171-186.

Krackhardt, D. (1988). "Predicting With Networks: Nonparametric Multiple Regression Analyses of Dyadic Data." Social Networks, 10, 359-382.

\section{See Also}

QAP

\section{Examples}

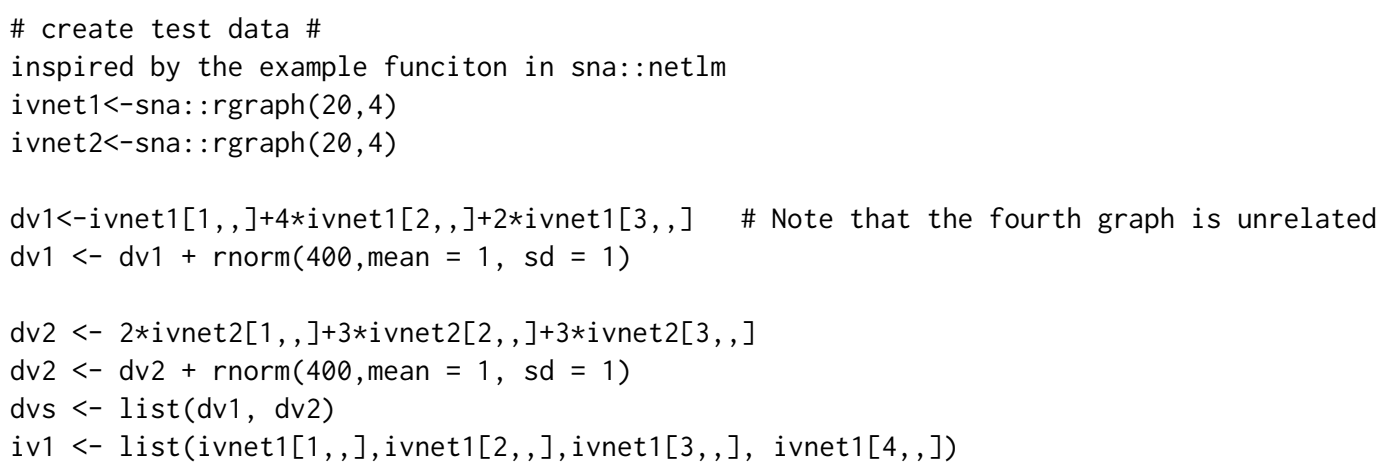


iv2 <- $\operatorname{list}(\operatorname{ivnet2}[1,],, \operatorname{ivnet2}[2,],, \operatorname{ivnet} 2[3,],, \operatorname{ivnet} 2[4,$,$] )$

ivs $<-$ list (iv1, iv2)

iv. names $=c($ "intercept", paste0 $(" I V ", 1: 4))$

QAP.MG (dvs, ivs, iv.names $=c($ "intercept", pasteø $(" I V ", 1: 4))$, samples $=3000)$ 


\section{Index}

QAP, 1,4

QAP.MG, 2, 3 